\title{
Le principe de diligence dans la procédure judiciaire sociale en Algérie
}

\section{Chakib Boukli Hacène}

\section{(2) OpenEdition}

1 Journals

\section{Édition électronique}

URL : https://journals.openedition.org/rdctss/1633

DOI : $10.4000 /$ rdctss. 1633

ISSN : 2262-9815

Éditeur

Centre de droit comparé du travail et de la sécurité sociale

\section{Édition imprimée}

Date de publication : 1 avril 2019

Pagination : 62-73

ISSN : 2117-4350

\section{Référence électronique}

Chakib Boukli Hacène, « Le principe de diligence dans la procédure judiciaire sociale en Algérie »,

Revue de droit comparé du travail et de la sécurité sociale [En ligne], 1 | 2019, mis en ligne le 01 novembre 2021, consulté le 13 novembre 2021. URL : http://journals.openedition.org/rdctss/1633 ; DOI : https:// doi.org/10.4000/rdctss. 1633

\section{(c) (†) $९$}

Revue de droit comparé du travail et de la sécurité sociale est mise à disposition selon les termes de la Licence Creative Commons Attribution - Pas d'Utilisation Commerciale - Pas de Modification 4.0 International. 


\section{LE PRINCIPE DE DILIGENCE DANS LA PROCÉDURE JUDICIAIRE SOCIALE EN ALGÉRIE}

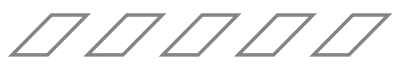

\section{RÉSUMÉ}

Suite à la promulgation du nouveau code algérien de procédure civile et administrative en 2008, l'Algérie a confirmé sa tendance à vouloir transformer la justice sociale d'une juridiction de droit commun respectant scrupuleusement la forme du procès civil, à une juridiction spéciale qui se rapproche dans la diligence de ses procédures et de son instance, aux juridictions du référé, bien qu'elle statue au fond, voire parfois même de manière définitive dès le premier degré de l'instance

MOTS CLÉS : Principe de diligence, justice du travail, procès social, décision de justice, juridiction du référé.

\section{ABSTRACT}

Following the promulgation of the new Algerian Code Civil and Administrative Procedure in 2008, Algeria has confirmed its tendency to transform the social justice from a common juridiction law that respects the form of civil lawsuit to a special juridiction warly closer in the diligence of its procedures and its instances to the summary juridiction, althrough it rules on the merits or sometimes even definitively in the first degree of the instance.

KEY WORDS : Principle of Diligence, Labor Justice, Social Lawsuit, Justice Decision, Summary Juridiction. 


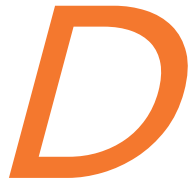

ès les premières années de l'Indépendance, l'Algérie a consacré ses premières lois aux procédures judiciaires civiles, ainsi qu'à l'organisation juridictionnelle de ses instances judiciaires. Cela traduisait une volonté réelle des pouvoirs publics du pays de mettre fin, dans ce domaine en particulier, à la continuité du droit de l'ancienne puissance coloniale. C'était une question de souveraineté ; l'Algérie indépendante, devait se prévaloir d'une organisation judiciaire propre, qui répondait aux impératifs d'un Etat nouvellement constitué, et qui concordait aussi avec son choix, de l'époque, pour l'idéologie socialiste.

Cette nouvelle organisation judiciaire s'était caractérisée par l'absence d'ordre juridictionnel d'exception, car elle se voulait simple et générale, au vu des faibles moyens, humains et matériels qui s'offraient à elle au lendemain de l'Indépendance. Dans cette perspective, les pouvoirs publics de l'époque, ont institué, en première instance, les Tribunaux, qui sont, selon l'article premier de l'ancien Code de procédure civile ${ }^{1}$, des juridictions de droit commun, ayant compétence pour connaître toutes les actions civiles, commerciales ou sociales, sous réserve de leurs compétences territoriales. Les jugements rendus en toutes matières par ces tribunaux en premier ressort pouvaient faire l'objet d'appel par devant les Cours de Justice. Enfin, en matière de cassation, la Cour Suprême avait l'entière compétence pour statuer sur les pourvois en cassation formés contre les arrêts et jugements rendus en dernier ressort par les cours et tribunaux de tous ordres.

Il est aisé de constater, que l'actuelle organisation juridictionnelle du pays, instituée par la loi n 08-09 du 25 février 2008 portant nouveau Code de procédure civile et administrative, ainsi que la loi organique $n^{\circ} 05-11$ du 17 juillet 2005 relative à l'organisation judiciaire, ne présente pas de grandes disparités avec l'ancienne organisation. Néanmoins, il est à signaler que les pouvoirs publics ont opéré un important changement sur l'ordre juridictionnel de la période socialiste qui a sévi jusqu'à la fin des années quatre-vingts du siècle dernier. En effet, durant cette période l'organisation juridictionnelle ne reposait pas sur une stricte séparation entre la justice civile et administrative. La dualité de juridiction en Algérie, ne fut concrétisée qu'avec l'adoption de la Constitution de 1996 et la création en 1998 des tribunaux administratifs, le Conseil d'Etat et enfin le Tribunal des conflits.

Au sein de cette organisation judiciaire civile, dite ordinaire du fait qu'elle ne comporte pas de juridictions d'exception, il existe des instances propres à chaque contentieux, et à tous les niveaux de l'instance. Ce sont les sections au niveau du tribunal, alors qu'au niveau des Cours d'appel et de la Cour suprême, il y a des chambres.

Le contentieux social n'est pas en reste, car toutes les juridictions judiciaires algériennes, et de tous temps, ont comporté en leur sein, des instances spécialisées dans le contentieux

1 Voir Ordonnance $n^{\circ}$ 66-154 du 6 juin 1966, portant Code de procédure civile (abrogée par la loi n ${ }^{\circ}$ 08-09 du 25 février 2008). 
social. Et au regard de ces dernières, seul le premier degré de juridiction (la section sociale du tribunal) constitue un intérêt pour la présente étude, en raison, d'une part, de la composition singulière de la formation de jugement spécialisée dans le règlement des litiges sociaux ${ }^{2}$, individuels et collectifs et, d'autre part, dans la diligence de ses procédures et de son instance, qui ont pour but de rendre la justice et ainsi faire rentrer les salariés dans leurs droits légitimes dans les plus brefs délais. Il est vrai que toute justice souffre forcément de lenteur excessive dû à l'accroissement du contentieux et à la complexification de la procédure, ce qui représente pour les travailleurs un grief considérable.

A cet effet, et dans la perspective d'apporter des remèdes à cette lenteur qui porte préjudice aux justiciables les plus fragiles (les salariés), l'Algérie a pris le parti de vouloir transformer la justice sociale d'une juridiction de droit commun respectant scrupuleusement la forme du procès civil, à une juridiction (spéciale) qui se rapproche dans la diligence de ses procédures et de son instance, aux juridictions du référé, bien qu'elle statue au fond, voire parfois même de manière définitive dès le premier degré de l'instance.

Il convient donc de mettre en exergue cette tendance de la justice du travail en droit procédurier algérien, qui tend à devenir un principe général de droit. Cela s'effectuera de deux manières différentes, la constatation, en premier lieu, de ce principe de diligence dans les instances qui statuent au fond (I). Ensuite, en deuxième lieu, faire la lumière sur les nouvelles prérogatives du juge social en matière de référé (II).

\section{I - LE PRINCIPE DE DILIGENCE DANS LES JURIDICTIONS SOCIALES STATUANT AU FOND}

La section sociale qui se trouve au sein des tribunaux de premier ressort, est composée selon le procédé de l'échevinage d'un magistrat de carrière assisté par deux assesseurs travailleurs et de deux assesseurs employeurs de manière paritaire ${ }^{3}$. Elle a, selon l'article 500 du Code de procédure civile et administrative, compétence exclusive dans sept matières différentes relatives aux contentieux individuels et collectifs du travail ainsi que celui de la sécurité sociale ${ }^{4}$. C'est ce qui fait d'ailleurs son originalité.

Mais en dehors de cette compétence matérielle et cette composition collégiale, la juridiction sociale du premier ressort reste assujettie, en règle générale, aux procédures du procès civil, s'agissant de la saisine du tribunal ou du déroulement du procès ou enfin de la prononciation du jugement et de son exécution. En effet, selon les termes de l'article 41 de la loi $n^{\circ}$ 90-04 du 06 novembre 1990 relative au règlement des conflits individuels

2 Voir dans ce sens, M. N.-E. Koriche, Droit du travail, les transformations du droit algérien du travail entre statut et contrat, relation d'emploi et de travail, une contractualisation relative, t. 1, O. P. U. 102009, p. 74.

3 Voir dans ce sens, M. N.-E. Koriche, op.cit., pp. 73-76; R. Ouadah, Conflits individuels et collectifs du travail dans le contexte dans réformes économiques en Algérie, éd. Dar Houma 2003 (en langue arabe), p. 53.

4 II est à remarquer, que cette énumération exhaustive des matières est imparfaite car ne pouvant couvrir tout le contentieux à caractère social. Il aurait été souhaitable pour le législateur algérien de se contenter d'une formulation plus large telle que prévue dans l'article 20 de la loi n 90-04 relative au règlement des conflits individuels de travail. 
de travail : "sauf les cas ou la présente loi en dispose autrement, sont applicables les dispositions de l'ordonnance ${ }^{\circ}$ 66-154 du 8 juin 1966 portant code de procédure civile $»^{5}$.

Alors même que ces dispositions restent effectives, cela n'a pas empêché les pouvoirs publics de doter la justice du travail de règles procédurières particulières, ayant comme principale caractéristique " la diligence ».En effet, le règlement judiciaire du contentieux social ne pouvait se contenter de procédures de droit commun, de par leur lenteur et leur complexité, et cela dans un souci de préserver les intérêts sociaux professionnels des travailleurs. Cette exception à la règle procédurière de droit commun a touché tout le processus du déroulement de l'action judiciaire entreprise par-devant la section sociale du tribunal, de la saisine du tribunal compétent $(A)$ jusqu'à l'exécution de la décision de justice (B).

\section{A - LA DILIGENCE DES PROCÉDURES DANS LA SAISINE DU TRIBUNAL ET LE DÉROULEMENT DE L'INSTANCE}

L'action sociale, comme toute autre action de droit commun, reste assujettie aux procédures de saisine et d'instance édictées par le Code de procédure civile et administrative algérien ${ }^{6}$. II faut, entre-autre, que les parties jouissent d'une qualité et d'un intérêt prévu par la loi ${ }^{7}$, et que le tribunal soit saisi par le dépôt au greffe d'une requête écrite, signée et datée du demandeur ou de son mandataire ou de son avocat en autant de copie qu'il y a de parties ${ }^{8}$, ainsi que le dépôt des conclusions des parties prenantes au procès soit fait de manière contradictoire?

En plus des exigences procédurales puisées du droit commun, la saisine de la section sociale du tribunal ainsi que le déroulement de son instance, sont régis par des normes d'exception, cherchant, par tout moyen, l'atténuation de la durée du procès.

\section{Les procédures particulières concernant la saisine de la section sociale du tribunal}

Il faut, tout d'abord, selon l'article 504 du Code de procédure civile et administrative algérien, porter l'action par devant la section sociale du tribunal compétent, dans un délai n'excédant pas six (6) mois, à compter de la date du procès-verbal de non-conciliation, sous peine de forclusion. En effet, le règlement des conflits individuels du travail est obligatoirement soumis, avant toute saisine du tribunal, à une tentative de conciliation effectuée par les bureaux de conciliation ${ }^{10}$. Et si jamais, le différend entre les parties

5 Pour rappel, l'Algérie dispose actuellement d'un nouveau Code de procédure civile instauré par la loi 08-09.

6 Voir l'article 1 du C. P. C. A. stipulant que : "les dispositions du présent code s'appliquent aux actions engagées devant les juridictions de droit commun ... ».

7 Voir l'article 13 du C.P. C. A.

8 Voir l'article 14 du C.P. C. A.

9 Voir l'article 263 du C. P. C. A.

10 Cette procédure préalable de conciliation reste facultative lorsque le défendeur réside en dehors du territoire national, ou en cas de faillite ou de règlement judiciaire de l'employeur. V. art. 19 de la loi $n^{\circ}$ 90-04. 
persiste, le bureau est contraint d'établir un procès-verbal de non-conciliation ${ }^{11}$. Dans ce cas, la partie ayant intérêt, munie de ce procès-verbal, peut saisir la section sociale du tribunal compétent.

Mais devant ce que les pouvoirs publics considèrent comme étant des abus en matière de demande d'introduction d'instance, où les parties en conflit, les travailleurs en général, préféraient attendre quelque mois, voire des années, avant de saisir le tribunal, recherchant ainsi un éventuel changement d'un juge ou d'un assesseur qui compose la section sociale du tribunal concerné, ou bien encore la cessation de l'activité légale de l'employeur pour pouvoir négocier directement avec le liquidateur entant que personne étrangère à l'entreprise, il a été exigé que l'action de justice soit inscrite et enrôlée par devant le greffe du tribunal dans un délai de six mois à compter de la délivrance du procès-verbal de nonconciliation, sous peine de forclusion.

Néanmoins, cette exigence reste inopérante, tant que la loi ne prévoit pas un délai de forclusion pour la saisine du bureau de conciliation. Car il est aisé aux parties litigieuses de ne présenter, par devant le bureau de conciliation, leur litige du travail, qu'après un certain temps.

Par ailleurs, lors de l'enregistrement de la requête d'introduction d'instance, le greffier se doit, en matière sociale, conformément aux articles 505 du Code de procédure civile et administrative et 38 de la loi relative au règlement des conflits individuels du travail, fixer la première audience au plus tard dans les quinze (15) jours qui suivent la date d'introduction de l'instance. Cela est considéré comme une réelle exception aux règles procédurières de droit commun qui ne fixe aucun délai particulier à la tenue de la première audience.

C'est d'autant plus vrai, que l'article 16 du Code de procédure civile et administrative, exige d'observer au moins un délai de vingt (20) jours entre la date de remise de la citation à comparaître et la date de la première audience. Ce délai est augmenté de trois (3) mois, si la personne citée à comparaître réside à l'étranger. Par conséquent, il est impossible, au vu du délai très court de la tenue de la première audience en matière sociale, de pouvoir faire bénéficier le défendeur des délais de comparution fixés par la loi. Il revient donc au juge de la section sociale et à ses assesseurs, d'estimer la suffisance du délai accordé au défendeur pour pouvoir se présenter à la première audience, sachant qu'il ne peut prétendre à un délai excédent les quinze (15) jours.

Dans la pratique, ce délai de quinze (15) jours n'est guère respecté, ni par les tribunaux qui fixent, en général, la date de la première audience au-delà des quinze (15) jours au vue du nombre élevé des affaires à traiter, ni par les huissiers de justice qui demandent toujours un délai supplémentaire afin de pouvoir réaliser la citation à comparaître. Dans ce cas de figure, où l'une des partie se trouve empêchée de comparaître pour défaut de citation, le juge peut, conformément à l'article 264 du Code de procédure civile et administrative, prolonger le délai de citation en renvoyant l'affaire à une prochaine audience. Ce problème de respect des délais fixés par la loi ne peut être réglé, qu'une fois que le législateur aura légiféré sur un délai de citation propre à l'action sociale, en concordance avec le délai de la tenue de la première audience.

11 Pour plus d'informations sur la composition et les prérogatives des bureaux de conciliation dans le cadre du règlement des contentieux du travail voir $\mathrm{M}$. N-E. Koriche, «A propos des procédures préalables de la saisine de la justice sociale en Algérie », Revue de droit comparé du travail et de la sécurité sociale, Comptrasec, 2014/2, p. 38 


\section{2 - Les procédures particulières concernant le déroulement de l'instance de} la section sociale du tribunal

Il s'agit là d'écourter autant que possible la durée des débats entre les parties au procès pour ne pas mettre à mal les droits sociaux professionnels des travailleurs. A cet effet, le juge, selon les articles 505 du Code de procédure civile et administrative et 38 de la loi relative au règlement des conflits individuels du travail, est tenu de statuer dans les plus brefs délais. Au sens de ces deux textes de loi, il est raisonnable de penser que le juge et ses assesseurs de la section sociale doivent se contenter de deux à trois audiences avant de clore les plaidoiries afin de délibérer.

\section{B - LES DÉCISIONS DE JUSTICE À CARACTÈRE DILIGENT PROPRES À LA SECTION SOCIALE}

A la lecture des textes de loi, on peut constater que le juge de la section sociale jouit d'un pouvoir décisionnel exceptionnel. Effectivement, il a la faculté de statuer en premier et dernier ressort par décision définitive ayant acquis l'autorité de la chose jugée, dans les cas prévus par la loi (1).Il lui est permis aussi, de rendre des décisions à exécution provisoire, voire même à exécution immédiate (2).

\section{1 - Les jugements de la section sociale rendus en premier et en dernier ressort}

L'article 21 de la loi relative au règlement des conflits individuels du travail donne compétence à la section sociale de statuer en premier et dernier ressort sur les matières suivantes:

- Lorsque la demande porte au principal sur l'annulation de sanctions disciplinaires édictées par l'employeur à l'encontre du demandeur travailleur, sans qu'il ait été fait application des procédures disciplinaires légales et/ou conventionnelles obligatoires ;

- Lorsqu'il s'agit de la délivrance de certificat de travail, bulletins de paie ou d'autres documents légalement prévus, pour attester de l'activité professionnelle du demandeur travailleur.

II faut ajouter à ces deux précédents cas, la sanction propre au licenciement abusif ${ }^{12}$, car survenant en violation des dispositions de l'article 73 de la loi $n^{\circ}$ 90-11 du 21 avril 1990, relative aux relations de travail, et prévue par l'article 73-4 alinéa 2 de la même loi. Conformément aux dispositions de cet article 73-4, la section sociale statue, là aussi, en premier et dernier ressort, en se prononçant soit sur la réintégration du travailleur dans l'entreprise avec maintien de ses avantages acquis, soit sur l'octroi au travailleur d'une compensation pécuniaire qui ne peut être inférieure à six (6) mois de salaire, sans préjudice des dommages et intérêts éventuels ${ }^{13}$.

12 En droit algérien le licenciement abusif est un licenciement à caractère disciplinaire qui n'est pas justifié par une faute grave commise par le travailleur. La qualification des fautes graves est allouée à la loi (art. 73 de la loi relative aux relations de travail) et au règlement intérieur de l'entreprise conformément à l'article 77 de cette même loi. Voir sur ce sujet L. Borsali Hamdan, Droit du travail, Berti Editions, Alger, 2014, p. 165.

13 Voir sur le licenciement irrégulier et abusif en Algérie, M. N.-E. Koriche, op. cit., p. 218. 
Par conséquent, si le licenciement disciplinaire est réputé abusif ou irrégulier, ou lorsque le travailleur se trouve empêché par le fait de son employeur, de prouver sa qualité professionnelle, la section sociale du tribunal compétent, statue en premier et dernier ressort ${ }^{14}$. Cela constitue une exception au " principe de la double juridiction " consacrée par l'article 6 du Code de procédure civile et administrative.

En effet, les juridictions de droit commun du premier degré, y compris la section sociale, statuent, généralement, en premier ressort, par jugements susceptibles d'appel, à moins que le montant des demandes, présentées par le demandeur, n'excède pas deux cent mille (200.000) dinars algériens. Dans ce cas, le tribunal statue en premier et dernier ressort ${ }^{15}$.

\section{2 - Les jugements ou les ordonnances de la section sociale à exécution provisoire ou immédiate}

L'exécution provisoire est définie par un auteur français comme étant « un bénéfice qui permet au gagnant d'exécuter un jugement dès sa signification, malgré l'effet suspensif du délai des voies de recours ordinaire ou de leur exercice ${ }^{16}{ }^{16}$. II s'agit donc d'un moyen rapide et exceptionnel, accordé à la partie au procès qui a eu gain de cause, afin de mettre en échec tout obstacle s'opposant à l'exécution de sa décision de justice.

En droit algérien, le juge et ses assesseurs formant la section sociale, jouissent de la faculté de rendre des décisions de justice à exécution provisoire dans des cas précisés par la loi de manière limitative. Cette règlementation des décisions de justice à exécution provisoire ou immédiate, recèle en son sein, des divergences et des contradictions qui sont de nature à rendre la mission du juge très ardue, voire parfois impossible.

En effet, selon l'article 22 de la loi relative au règlement des conflits individuels du travail, le juge et ses assesseurs formant la section sociale du tribunal compétent, sont dans l'obligation de rendre des décisions de justice à exécution provisoire, dans les matières suivantes:

- L'application ou l'interprétation d'une convention ou un accord collectif de travail ;

- L'application ou l'interprétation de tout accord conclu au titre de la procédure de conciliation devant le bureau de conciliation ;

- Le paiement des rémunérations et indemnités des six (6) derniers mois.

Les deux premiers cas constituent un moyen offert à l'une des parties, travailleur ou employeur, ayant intérêt pour faire valoir ses droits, de façon immédiate, à travers l'interprétation ou l'application, soit d'une convention ou d'un accord collectif de travail, soit d'un accord conclu au titre de la procédure de conciliation et figurant dans le procèsverbal de conciliation émanant du bureau de conciliation. II est vrai que l'exécution provisoire pour ces deux cas, demeure nécessaire pour garantir la force obligatoire des conventions ou accords collectifs et des accords de conciliation. Cela éviterait forcément,

14 Ce jugement est réputé définitif et ne peut être frappé d'appel. Cependant, il reste susceptible de pourvoi en cassation conformément à l'article 350 de Code de procédure civile et administrative

15 Voir article 33 du C. P. C. A.

16 J. Vincent, Procédure civile, 19 ème éd., Précis Dalloz 1978, p. 739, nº 561. 
la survenance éventuelle de litiges entre les parties signataires, ou pis encore, qu'un litige aux conséquences limitées, se dégénèrerait en un grand conflit collectif.

Concernant le troisième cas de jugement à exécution provisoire, en l'occurrence le paiement des rémunérations et indemnités des six (6) derniers mois, le bénéficiaire réside forcément en la personne du travailleur. A cet effet, le juge et ses assesseurs se doivent de statuer avec diligence, sur le bien-fondé de la demande du travailleur, et ainsi rendre un jugement à exécution immédiate nonobstant appel ou opposition, en raison du caractère alimentaire du salaire. Par contre, pour les rémunérations et indemnités qui vont au-delà des six (6) derniers mois, leur exécution provisoire n'est pas obligatoire. Elle reste soumise à l'appréciation du juge et des assesseurs formant la section sociale ${ }^{17}$. De plus la demande de créance concernant les salaires se prescrit, conformément à l'article 309 du Code civil, par cinq ans. Cela veut dire, que le travailleur ne peut entreprendre une action de justice pour réclamer des salaires non payés vieux de cinq ans et plus.

On en déduit, que la section sociale formée par un juge professionnel et des assesseurs représentant les travailleurs et les employeurs, rend par jugement en premier ressort, de manière obligatoire ou facultative selon les cas prévus par la loi, des décisions à exécution provisoire. Par conséquent, la loi relative au règlement des conflits individuels du travail dans son article 22, accorde ce pouvoir au juge et aux assesseurs formant la section sociale. C'est donc une compétence collégiale qui s'exerce au moyen d'un jugement.

Néanmoins, et en ce qui concerne l'exécution du procès-verbal de conciliation qui émane du bureau de conciliation, l'article 34 alinéa 1 1er de la même loi édicte qu'« en cas d'inexécution de l'accord de conciliation par l'une des parties dans les conditions et les délais fixés à l'article 33 de la présente loi, le président du tribunal, siégeant en matière sociale, saisi d'une requête à exécution, ordonne à sa première audience, le défendeur régulièrement convoqué, l'exécution immédiate du procès-verbal de conciliation, sous astreinte journalière qui ne peut être inférieure à $25 \%$ du salaire mensuel minimum garanti, tel que fixé par la législation et la règlementation en vigueur ». A la lecture de ce texte, on peut constater que la même loi recèle en son sein deux dispositions contradictoires.

En effet, l'article 34, suscité, donne compétence des décisions de justice à exécution immédiate et non pas provisoire, bien que cela soit la même chose, concernant l'exécution du procès-verbal de conciliation, à la seule personne du président du tribunal siégeant en matière sociale (la section sociale), sans ses assesseurs. C'est donc une compétence individuelle et non plus collégiale comme prévu par l'article 22. Par ailleurs, la décision à exécution immédiate est exercée par ordonnance et non plus par jugement, chose réservée d'ordinaire au juge du référé.

Cette contradiction est de nature à compliquer la tâche du juge qui se retrouve embarrassé, ne sachant pas vers à quelle disposition se référer. Lui faudrait-il privilégier la rapidité et l'efficacité en optant pour les dispositions de l'article 34 qui lui offrent les prérogatives du juge du référé et la possibilité d'ordonner l'exécution immédiate, sous astreinte, du procès-verbal de conciliation dès la première audience, privant ainsi le défendeur, bien qu'il soit régulièrement convoqué (cité à comparaître), de faire valoir ses exceptions et d'être entendu par le juge contradictoirement. Ou bien opterait-il pour les dispositions de l'article 22, en prenant plus de temps à décider de l'exécution provisoire,

17 Voir article 22 de la loi $n^{\circ} 90-04$ relative au règlement des conflits individuels du travail. 
après qu'il ait pu entendre les parties contradictoirement, et qu'il ait délibéré collégialement en présence de ses assesseurs par jugement?

Pour mettre fin à cette divergence normative, le législateur est intervenu en 2008 à travers la promulgation du nouveau Code de procédure civile et administrative. En effet, il est stipulé dans son article 508 que: «le président de la section sociale peut être saisi par requête aux fins d'exécution immédiate, dans les deux cas suivants :

- inexécution de l'accord de conciliation par l'une des parties ;

- inexécution de tout ou partie d'un accord collectif de travail auquel sont parties des représentants de travailleurs et un ou plusieurs employeurs ».

Aussi l'article 509 du Code de procédure civile et administrative, prévoit que "le président de la section sociale ordonne l'exécution immédiate de la décision, sous astreinte comminatoire, telle que prévue par la législation du travail. L'ordonnance est exécutoire de plein droit, nonobstant l'exercice de toutes voies de recours ".

Il est à remarquer, que ces deux articles du Code de procédure civile et administrative, ne prévoient l'exécution immédiate que dans deux cas seulement. Le premier concerne l'inexécution d'un accord de conciliation émanant du bureau de conciliation, où le législateur du nouveau code, a repris la même formulation de l'article 34 de la loi relative au règlement des conflits individuels du travail, à l'exception de l'obligation faite au président de la section sociale, d'ordonner dès la première audience l'exécution immédiate du procès-verbal de conciliation. Quant au deuxième cas, il porte sur l'inexécution d'un accord collectif de travail prévu par l'article 35 de la même loi, s'agissant de l'exécution sous astreinte seulement. Cela veut dire que le juge de la section sociale se trouve, là aussi, investi des prérogatives du juge du référé.

Par conséquent, il est aisé de constater que le nouveau Code de procédure civile et administrative adopté en 2008 a privilégié les dispositions des articles 34 et 35 de la loi de 1990 relative au règlement des conflits individuels du travail, en accordant au juge de la section sociale les prérogatives du référé. II a aussi abrogé implicitement et partiellement les deux premiers cas prévu par l'article 22 de cette même loi, à savoir, l'inexécution du procès-verbal de conciliation relative au règlement des conflits individuels du travail et de l'accord collectif de travail. L'abrogation implicite a pour fondement l'alinéa 3 de l'article 2 du Code civil, du fait que le nouveau texte règlemente différemment une matière auparavant régie par l'ancien texte.

Mais en dépit de son abrogation partielle et implicite, l'article 22 de la loi relative au règlement des conflits individuels du travail, conserve la majorité de ses dispositions, en l'occurrence, son troisième cas, dans sa totalité, relative au non payement des six derniers mois de salaire et des indemnités professionnelles. Quant aux deux premiers cas, règlementés de nouveau par le Code de procédure civile et administrative, ils continuent d'être régis par l'article 22 de cette même loi pour tout ce qui est relatif à l'interprétation des procès-verbaux de conciliation et des conventions et accords collectifs de travail.

Enfin, eu égard à la formulation maladroite de l'article 508 du Code de procédure civile et administrative, qui restreint ses dispositions au seul accord collectif de travail auquel sont parties des représentants de travailleurs et un ou plusieurs employeurs, il est permis de dire que les conventions collectives de travail, qui constituent selon l'article 114 de loi relative aux relations de travail, un accord écrit sur l'ensemble des conditions d'emploi et de travail pour une ou plusieurs catégories professionnelles, restent assujetties aux dispositions de 
l'article 22 de la loi relative aux règlements des conflits individuels du travail, du fait de ne pas avoir été abrogées implicitement par l'article 508 du Code de procédure civile et administrative.

\section{II - LE PRINCIPE DE DILIGENCE DANS LES JURIDICTIONS DU RÉFÉRÉ}

En cas de situation urgente ou pour des difficultés d'exécution ayant trait aux relations collectives ou individuelles du travail, les parties en conflit ont le droit de saisir le juge du référé afin d'ordonner les mesures adéquates et nécessaires pour y remédier de manière diligente. Ces prérogatives du juge du référé sont en droit social algérien allouées d'une part, au président du tribunal qui jouit de ces attributions de manière exclusive (A). D'autre part, et de manière exceptionnelle, au juge de la section sociale du tribunal compétent (B).

\section{A - LES ATTRIBUTIONS DU PRÉSIDENT DU TRIBUNAL (DU RÉFÉRÉ) DANS LE CONTENTIEUX SOCIAL}

Comme le fait remarquer un auteur algérien, il n'existe pas un juge du référé propre au contentieux social ${ }^{18}$. Et en cas d'urgence ou de difficulté d'exécution les parties peuvent saisir le président de la section des référés qui a une compétence générale dans ce domaine. Pour cela, il faudra aux parties en conflit, les travailleurs et les employeurs, se conformer aux dispositions du Code de procédure civile et administrative dans son chapitre réservé au référé et aux ordonnances de référé. Effectivement, l'action en référé est assujettie à deux sortes de condition, des conditions procédurales (1) et des conditions de fond (2).

\section{1 - Les conditions procédurales de l'action en référé}

Selon l'article 299 du Code de procédure civile et administrative, dans tous les cas d'urgence, ou lorsqu'il s'agit de décider d'une mesure de séquestre ou de toute mesure conservatoire, l'affaire est portée par devant la section des référés au sein du tribunal du lieu de l'incident ou de la mesure sollicitée, au moyen d'une requête écrite d'introduction d'instance remise par le demandeur ayant intérêt et qualité, au greffe du tribunal qui se charge de l'enregistrer et de fixer la date de la première audience, dans les plus brefs délais, vu l'urgence.

Aussi, les délais de citation peuvent être réduits au maximum à vingt-quatre heures ; mais en cas d'extrême urgence la citation peut avoir lieu d'heure à heure, à condition qu'elle soit signifiée en personne au défendeur ou à son représentant légal ou conventionnel ${ }^{19}$. Dans ce cas il est permis de présenter la requête directement, et en dehors des heures de travail et les jours fériés, au magistrat chargé des référés au siège de la juridiction, avant inscription sur le registre tenu au greffe ${ }^{20}$.

18 M. N.-E. Koriche, op.cit., p. 78.

19 Voir article 301 C. P. C. A.

20 Voir article 302 C. P. C. A. 


\section{2 - Les conditions de fond de l'action en référé}

L'action en référé constitue une procédure exceptionnelle instituée dans les cas d'urgences ou de mesure de séquestre ou de toute mesure conservatoire. Elle requière une procédure contradictoire, contrairement aux ordonnances sur requête ${ }^{21}$. Par principe, et conformément à l'article 303 de Code de procédure civile et administrative, l'ordonnance de référé ne préjudicie pas au principal, elle est prévue, plutôt, pour permettre au demandeur de parer au plus pressé en faisant prendre, par une voie rapide, des mesures immédiatement exécutoires, mais de caractère provisoire, nonobstant les voies de recours ${ }^{22}$.

\section{B - LES ATTRIBUTIONS DU PRÉSIDENT DE LA SECTION SOCIALE EN MATIÈRE DE RÉFÉRÉ}

La section sociale est une juridiction de fond, elle statue au principal afin de mettre fin au litige à caractère social. A cet effet, dans le cas où le litige ne constitue pas une contestation sérieuse au fond, son règlement est soumis exclusivement à la section des référés qui siège dans le même tribunal compétent. Exceptionnellement, le président de la section sociale du tribunal du premier ressort ainsi que le président de la chambre sociale se trouvant au niveau de la Cour d'appel, se voient attribuer les prérogatives du juge des référés, mais seulement dans un domaine très restreint.

Effectivement, et selon les articles 506 et 507 du Code de procédure civile et administrative, le président de la section sociale peut ordonner, par ordonnance susceptible d'appel, toutes mesures provisoires ou conservatoires, pour faire cesser tout acte de nature à entraver la liberté de travail. C'est dans ce seul domaine, où il est permis au juge de la section sociale de se prévaloir des attributions du juge des référés.

A la lumière des articles 34, 35 et 36 de la loi n 90-02 du 06 février 1990, relative à la prévention et au règlement des conflits collectifs de travail et à l'exercice du droit de grève, l'entrave à la liberté du travail se réalise par des actes de nature à empêcher, par menaces, manœuvres frauduleuses, violence ou voies de fait, un travailleur, un employeur ou ses représentants, d'accéder à leur lieu habituel de travail, de reprendre ou de poursuivre l'exercice de leur activité professionnelle. Dans ces cas précis, le demandeur peut saisir directement la section sociale du tribunal compétent, afin de lui permettre de parer au plus pressé, en faisant prendre par ordonnance susceptible d'appel, des mesures provisoires ou conservatoires à caractère exécutoire.

Si jamais l'occupation des locaux professionnels, par des travailleurs grévistes, avait pour but d'entraver la liberté du travail telle que prévue par l'article 34 de la loi relative à la prévention et au règlement des conflits collectifs de travail et à l'exercice du droit de grève, le président de la section sociale, peut ordonner l'évacuation des locaux, conformément à l'article 35 de la même loi, sur demande de l'employeur.

En dépit de ces attributions exceptionnelles allouées au président de la section sociale, il aurait mieux fallu les élargir, au point de permettre à cette juridiction du fond de statuer en la forme du référé, dans tous les cas d'urgence ou pour décider de toutes les mesures conservatoires ou de séquestre en relation avec les conflits individuels ou collectifs du travail. Cela aboutirait forcément à priver le juge du référé (le président du tribunal) de ses

21 Voir article 310 C.P. C. A. et s..

22 Voir article 303 C.P. C. A. 
prérogatives à chaque fois que le litige est à caractère social. En revanche, le président de la section sociale, étant un magistrat professionnel et jouissant d'une expérience indéniable dans le règlement des conflits sociaux, aurait dans ce type de litige une compétence exclusive.

En conclusion, il est avéré que le règlement particulier des procédures propres au procès social, édicté par la législation procédurale algérienne, tel que démontrée plus haut, demeure en totale concordance avec l'ordre public social qui a pour but la défense des intérêts des travailleurs, partie la plus vulnérable dans la relation de travail. En effet, il résulte de tout conflit social, individuel ou collectif, que le travailleur se retrouve, en général, en situation d'extrême précarité, particulièrement quand il y a perte d'emploi. Il était, par conséquent, impératif pour le législateur algérien, de prévoir une procédure judiciaire particulière, qui protège, par sa diligence, les intérêts des travailleurs.

Néanmoins, cette règlementation particulière n'est pas exempte de tout défaut, du fait qu'elle est limitée et restreinte, car ne concernant qu'une infime partie des procédures qui régissent le procès social. Ce dernier reste effectivement, gouverné, dans sa grande majorité, parle droit commun de la procédure civile, qui est inadapté à ce cas de figure. A cet effet, il serait préférable pour législateur algérien qu'il aille vers une généralisation totale de ses procédures particulières, qui se fondent essentiellement sur le principe de diligence, en les élargissant à tout le processus du déroulement de l'action sociale, depuis la saisine de la juridiction compétente jusqu'à l'exécution de la décision de justice.

Par ailleurs, il est aussi regrettable de voir un réel décalage entre ce qui est prévu dans les textes de loi en matière de procédures diligentes, et son application par les personnes concernées. En effet, et en dépit de toutes les dispositions normatives cherchant à affirmer le principe de diligence dans la procédure du procès social, les sections sociales des tribunaux se contentent généralement d'appliquer les procédures judiciaires civiles communes à tout procès, quelle que soit sa nature, malgré leur lenteur, privant ainsi le justiciable (le travailleur en particulier), des avantages et exceptions que lui confère la loi en matière sociale.

\section{CHAKIB BOUKLI-HACÈNE}

Professeur, Faculté de droit et des sciences politiques. Université Dr. Moulay Tahar de Saïda.

Thèmes de recherche : Droit syndical, droit de l'emploi, les normes RSE et le droit social algérien, la flexibilité du contrat de travail.

\section{Publications:}

C. Boukli-Hacène, "Le principe de faveur en droit du travail et les normes RSE ", Revue académique de la recherche juridique, Faculté de droit et des sciences politiques, Université de Béjaia, nº́spécial 2015.

C. Boukli-Hacène, "Le débauchage, une pratique commerciale déloyale assujettie à la législation du travail ", Revue des recherches juridiques et politiques, Faculté de droit et des sciences politiques, Université de Saida, $n^{\circ}$ 10/2018.

C. Boukli-Hacène, "L'apport de la loi de finances 2018 au droit social algérien ", Revue de droit comparé du travail et de la sécurité sociale, Comptrasec, n 1/2018. 